\title{
Historical Events in Neuroimaging and Vascular Intervention in Sree Chitra Tirunal Medical Centre, Thiruvananthapuram
}

\author{
Marthanda Varma Sankaran Valiathan ${ }^{1}$ \\ ${ }^{1}$ Manipal School of Life Sciences, Manipal Academy of Higher \\ Education (MAHE), Manipal, Karnataka, India
}

J Clin Interv Radiol ISVIR 2021;5:70-71.

\section{Neuroradiology}

The historical events in neuroimaging and vascular intervention narrated by Vedula Rajainikanth Rao and Ravi Mandalam Kolathu in the current issue ${ }^{1,2}$ took place in the erstwhile Sree Chitra Tirunal Medical Centre (Chitra), Thiruvananthapuram. In the 1970s, it was a hospital with 120 beds under the Government of Kerala, but stood apart by being sponsored by the Department of Science and Technology of Kerala, mandating the hospital to provide clinical inputs for medical technology development from their services, which were offered only to referred cardiology and neurology patients (-Fig. 1). The hospital opened its doors to patients in 1976 and Professor Mahadevan Pillai joined as head of the Radiology Department. A pioneer who had trained under masters like Seldinger played a major role as a founder of neuroradiology while serving in the Barnard Institute, Chennai, and National Institute of Mental Health and Neurosciences (NIMHANS), Bangalore. He was a brilliant radiologist, innovator, and a splendid colleague whose friendliness was infectious! VRK Rao joined him in 1977 and Ravi Mandalam soon after. Their main equipment was a Diagnost 77, Philips unit for myelography, and general-purpose radiography. I remember Professor Pillai's unique description of an in-house designed and built manual changer of cassettes for serial angiography. Rao was a quick learner and soon became the highly skilled partner of Professor Pillai. To perform percutaneous carotid angiography with our equipment demanded ingenuity. It was indeed daring and was further tested when simultaneous injections of both carotid arteries were done. This obtained images in the axial view, enabling assessment of the lateral extent of intrasellar pituitary tumors. This was of special interest to Professor George Mathew who greatly valued the contributions of neuroradiology to expand his transsphenoidal approach to the pituitary. The angiographic

\footnotetext{
Address for correspondence Prof Marthanda Varma Sankaran Valiathan, ChM, FRCS, FRCS (C), FRCP, DSc (h.c), Manipal School of Life Sciences, Manipal Academy of Higher Education (MAHE), Dr. TMA Pai Planetarium Complex, Manipal 576104, Karnataka, India (e-mail: msvaliathan@gmail.com).
}

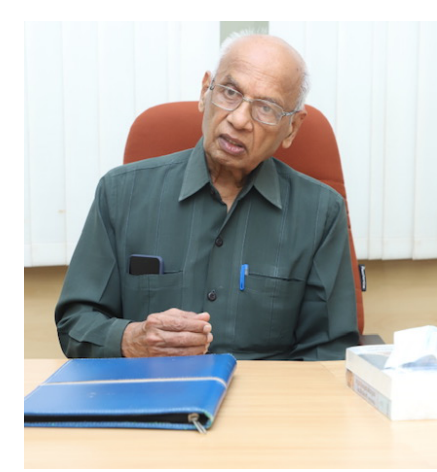

procedures were improved by locally developed methods of magnification and subtraction, which were a notable example of frugal innovation or Indian jugaad! Soon a Mimer III-Elema Schonander-was added to the armamentarium, which became the workhorse of angiography and tomography. This was followed by a $70 \mathrm{~mm}$ camera, roll film, and a Newton Hastystereotome. As the power of machines grew, human mind kept pace as Professor Pillai taught Rao how to obtain stereoscopic vision by training the position of both eyes while focusing on the object of interest to bring out a third image! Mimer III made pneumoencephalography a routine procedure. The addition of cerebral ventriculography using Conray 280, myelography employing Amipaque, and air myelography expanded the range of diagnostic studies in Chitra, which attracted the favorable notice of peers and drew patients in increasing numbers to the hospital.

Rao's description of balloon angioplasty being preferred to surgical angioplasty of subclavian artery is etched in my mind. It reminded me of John Hunter's prescient observation in the 18th century that a surgical operation is "like an armed savage trying to get by force what a civilised man would get by stratagem." It was natural that interventional radiology grew rapidly at Chitra with the energetic pursuit of new stratagems by Rao and Ravi Mandalam. Occlusive disease of peripheral arteries caused by atherosclerosis in the old and by aortoarteritis in the young underwent balloon angioplasty. The Chitra team acquired valuable experience and learnt important lessons that were reported in scientific journals. Their appetite for novel techniques was insatiable. An example was the effort to develop neodymium-doped yttrium aluminum garnet laser to bore a channel in a totally occluded artery, followed by balloon angioplasty. Though this was done successfully in experimental animals and one patient, the technique was soon superseded by better methods. Ravi Mandalam went further and took the initiative to develop implantable “

DoI https://doi.org/ $10.1055 / \mathrm{s}-0041-1731132$ ISSN 2457-0214
(C) 2021. Indian Society of Vascular and Interventional Radiology.

This is an open access article published by Thieme under the terms of the Creative Commons Attribution-NonDerivative-NonCommercial-License, permitting copying and reproduction so long as the original work is given appropriate credit. Contents may not be used for commercial purposes, or adapted, remixed, transformed or built upon. (https://creativecommons.org/licenses/by-nc-nd/4.0/).

Thieme Medical and Scientific Publishers Pvt. Ltd. A-12, 2nd Floor, Sector 2, Noida-201301 UP, India 


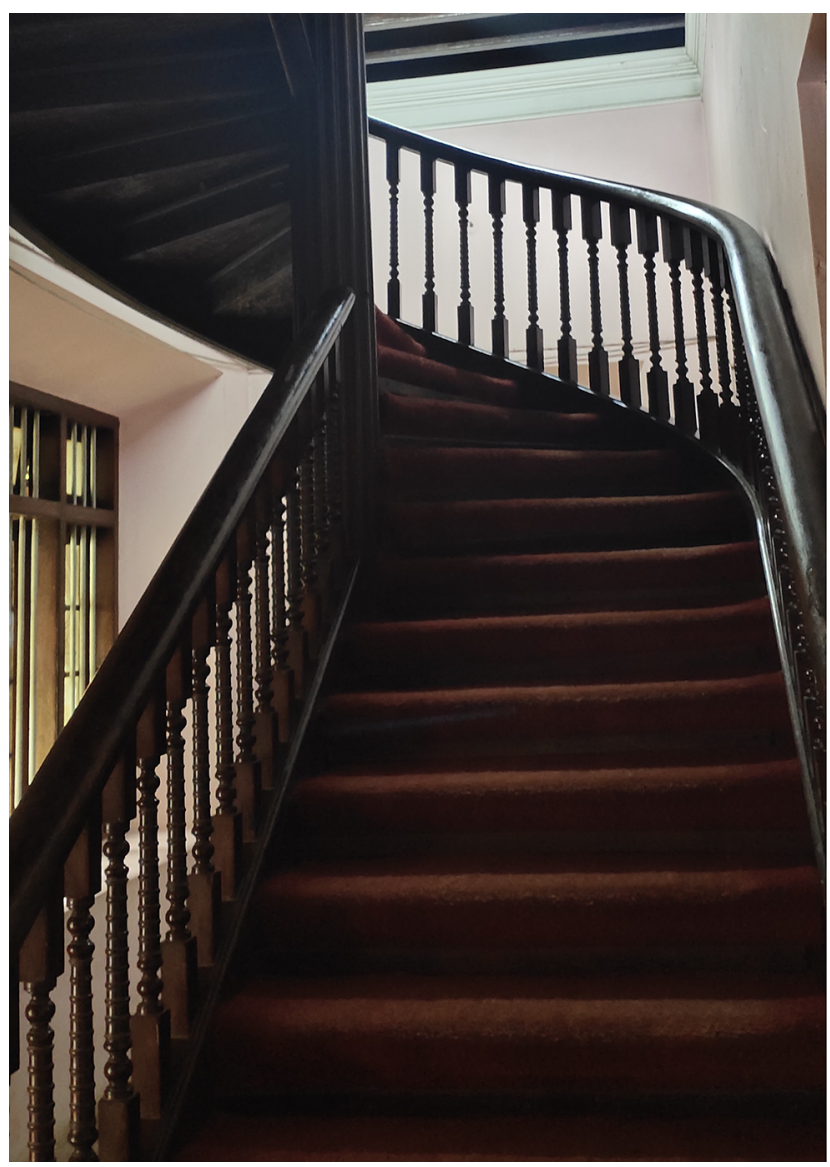

Fig 1. A beautiful wooden staircase in the Satelmond Palace, gifted by His Excellency Marthanda Varma for the research and development at the institute. Image courtesy: Mr Joy Vithayathil, Senior Librarian, SCTIMST, Trivandrum.

spring coils" (anticipating stents) and the team even made efforts to develop a "self-expandable stent."

\section{Interventional Neuroradiology}

In part II, the authors give a detailed account of their effort to develop interventional neuroradiology. Angiography had already shown that cerebral aneurysms were common and the presence of two neurosurgeons-Professors George Mathew and Damodar Raut-who had special interest in the treatment of aneurysms attracted increasing number of patients. Arteriovenous malformations (AVMs) were less frequent and Professor Mathew agreed with Rao that large AVMs would be suitable for management by embolotherapy using crushed muscle, dura, or silastic microspheres developed by Dr. Jayakrishnan of the Technology Wing of Chitra Institute. The techniques were successfully developed, used, carefully followed up, and later reported. French Professors Lucien Picard and Jack Moret were deeply impressed by the ongoing work at Chitra and provided crucial assistance in initiating endovascular treatment for AVMs, additionally donating catheter delivery systems. The successful management of large AVMs was performed side by side with super selective angiography of the branches of middle cerebral and other crucial arteries for occlusion. This would block the feeders to the AVM and facilitate surgery with reduced blood loss. More than 150 superselective procedures were done with partial obliteration of the AVM and radioanatomic cure obtained in more than $50 \%$ of patients.

\section{Postdoctoral Training}

The entire program in vascular and neuroradiology provided an opportunity for training in the new discipline of interventional radiology. A 1-year Post Doctoral Certificate Course was initiated in Chitra Institute with excellent response. Those who underwent this training serve across India, occupying high faculty positions and heading departments in major hospitals.

\section{Conclusion}

Sree Chitra Tirunal Medical Centre became an Institute of National Importance by an Act of Parliament in 1980 to promote the joint culture of medicine and technology for advancing health care. While its hospital sought to promote high quality service, it was also mandated to deliver clinical inputs for developing instruments and devices indispensable for the practice of medicine. The historical events at Chitra demonstrated that the joint culture of medicine and technology is the precursor of new instruments and devices, currently imported at high cost. To make high technology devices affordable and accessible to common people, pursuit of frugal innovation as outlined in these papers is necessary, inescapable, and commendable.

\section{Conflict of Interest \\ None declared.}

\section{Editors' note}

Prof Marthandavarma Sankaran Valiathan was awarded the Padma Vibushan, second highest civilian award of the Republic of India in 2005 for his contributions to health technology.

\section{References}

1 Vedula RR, Kolathu RM. Birth and Growth of Neuroimaging and Vascular Intervention at Sree Chitra Tirunal Institute for Medical Sciences \& Technology, Thiruvananthapuram - Part I. Clinical Interventional Radiology 2021

2 Vedula RR, Kolathu RM. Birth and Growth of Neuroimaging and Vascular Intervention at Sree Chitra Tirunal Institute for Medical Sciences \& Technology, Thiruvananthapuram - Part II. Clinical Interventional Radiology 2021 